\title{
Essais
}

Revue interdisciplinaire d'Humanités

14 | 2018

Plurilinguismes en construction

\section{Pour une méthodologie de la proximité ?}

Les enquêtes de terrain en sociolinguistique et didactique des langues

Marielle Rispail, Marine Totozani et Valeria Villa-Perez

\section{CpenEdition}

Journals

Édition électronique

URL : http://journals.openedition.org/essais/359

DOI : 10.4000 /essais.359

ISSN : 2276-0970

Éditeur

École doctorale Montaigne Humanités

Édition imprimée

Date de publication : 1 juin 2018

Pagination : 137-153

ISBN : 979-10-97024-06-2

ISSN : $2417-4211$

Référence électronique

Marielle Rispail, Marine Totozani et Valeria Villa-Perez, «Pour une méthodologie de la proximité ? », Essais [En ligne], 14 | 2018, mis en ligne le 01 décembre 2019, consulté le 12 décembre 2019. URL: http://journals.openedition.org/essais/359 ; DOI : 10.4000/essais.359 


\title{
Pour une méthodologie de la proximité ? \\ Les enquêtes de terrain en sociolinguistique et didactique des langues
}

\author{
Marielle Rispail, Marine Totozani, Valeria Villa-Perez
}

\section{Introduire l'idée de « proximité »... ${ }^{1}$}

En interrogeant une hypothèse du sociologue Pierre Paillé à propos des approches qualitatives considérées comme des "méthodologies de la proximité ", nous développerons dans cet article une réflexion sur ses manifestations/réalisations en sociolinguistique et en sociodidactique des langues. Une telle méthodologie prend place dans :

" une logique de proximité [qui] s'exerce au plus près des phénomènes qu'elle souhaite mettre en lumière, des acteurs qui les incarnent, des contextes qui les portent, mais aussi du chercheur qui les examine avec toute sa sensibilité théorique et expérientielle²."

Selon Pierre Paillé, cette logique est le trait commun à toutes les approches qui se réclament de la lignée qualitative. De façon plus détaillée, cette proximité est à entendre dans le sens de proche:

- de la vie dans sa complexité et sa mouvance telle qu'elle se donne et s'appréhende ;

- de l'expérience telle qu'elle peut être directement observée en dehors de toute manipulation ;

- de la parole, de son énonciation, de son organisation en action ;

- des contextes immédiats des expériences ou des phénomènes étudiés ;

- du sens des expériences tel qu'il peut être appréhendé ou construit ;

- du vécu des personnes dans leur monde intime, social ou culturel ;

- de la subjectivité du chercheur;

- des témoignages recueillis et des observations amassées qui sont analysées en l'état ;

\section{Nous remercions Rosa Pugliese pour sa relecture et la richesse de nos échanges.}

2 Pierre Paillé, "Les conditions de l'analyse qualitative. Réflexions autour de l'utilisation des logiciels ", in Sociologies, La recherche en actes, Champs de recherche et enjeux de terrain, mis en ligne le 06 juillet 2011, consulté le 22 juin 2017 : http://sociologies.revues.org/3357. 
- des interactions à travers lesquelles se construit la réalité ;

- des phénomènes étudiés, qui sont mis en récit et parfois racontés directement. ${ }^{3}$

La réflexion sur le concept de "proximité " a été menée jusqu’ici dans le champ des sciences humaines et sociales mais aucune recherche ne s'est occupée, à notre connaissance, de l'intérêt d'aborder les phénomènes langagiers à l'école et dans la société sous cet angle et dans une perspective sociolinguistique et sociodidactique. En effet, l'étude des phénomènes langagiers se singularise par la difficulté à cerner un objet par essence fluide, changeant, mobile, caractérisé par sa circulation dans le temps et l'espace. C'est en l'observant "à la loupe ", au plus près de ses émergences et de ses nuances, qu'on pourra peut-être rendre compte de sa dynamique et de ses évolutions. Cela suppose qu'on ne cherche ni à réduire leur subtilité, ni à généraliser nos résultats. Une certaine honnêteté éthique demande aussi qu'on fasse appel à la " solidarité scientifique » en favorisant les rencontres avec d'autres champs de recherche que le nôtre (les Sciences du langage), s'ils peuvent nous aider à éclairer les phénomènes interactifs par lesquels on appréhende les langues dans leur manifestation sociale. On en verra quelques exemples dans la deuxième partie de ce texte.

Étudier au plus près la vie du langage dans les situations scolaires et sociales qui sont nos terrains d'observation demande aussi qu'on s'interroge au préalable sur la notion de "terrain $"^{4}$ que rencontre d'emblée notre tentative de proximité. On est en effet pris par la double exigence de ne pouvoir étudier les phénomènes langagiers sans référence au "terrain " tout en rencontrant de grandes difficultés à le définir. Qu'est-ce qu'un " terrain de recherche " pour qui étudie la vie des langues? Que veut dire "faire du terrain " ? La question soulève des interrogations méthodologiques et éthiques, comme le soulignent Véronique Fillol et Philippe Le Meur ${ }^{5}$ et invite le/la linguiste à regarder du côté de l'ethnologie, de l'anthropologie et des enquêtes ethnographiques. Loin d'une vision simplificatrice qui voudrait séparer le terrain " concret » des réflexions que peut susciter sa description, nous nous inscrivons dans la conception dialectique qui propose que le terrain, dans ses dimensions géographique, temporelle et sociale, intègre le/la chercheur.e dans un " réseau d'interactions humaines et sociales ${ }^{6}$ et d'influences réciproques qui interdisent finalement

3 Pierre Paillé, "La recherche qualitative une méthodologie de la proximité », in Henri Dorvil (éd.), Problèmes sociaux. Théories et méthodologies de la recherche, t. 3, Québec, Presses des Universités de Québéc, 2007, p. 432-433.

4 Marielle Rispail, «Terrain », in Marielle Rispail (éd.), Abécédaire de Sociodidactique. 65 Concepts et notions, Saint-Étienne, PUSE, 2017, p. 122-123.

5 Véronique Fillol et Philippe Le Meur, "Introduction : enquête de terrain et décolonisation dans le Pacifique sud. Science, politique, éthique ", in Véronique Fillol et Philippe Le Meur (éds), Terrains Océaniens : enjeux et méthodes, Paris, L'Harmattan, 2014, p. 9-26.

6 Philippe Blanchet, "Nécessité d'une réflexion épistémologique ", in Patrick Chardenet (éd.), Guide pour la recherche en didactique des langues et des cultures. Approches contextualisées, Paris, Les archives contemporaines, 2015, p. 13. 
de postuler qu'il pré-existerait à toute recherche, et imposent au contraire l'idée que le/la chercheur. $\mathrm{e}^{7}$ construit son terrain autant que le terrain construit le/la chercheur.e. Dans ce sens, Laplantine ${ }^{8}$ écrivait déjà en 1996 : " Il n'existe donc pas, à proprement parler de données ethnographiques, mais d'emblée partout et toujours (...) l'interaction entre un chercheur et ceux qu'il étudie. C'est précisément cette rencontre qui mérite d'être appelée "terrain" ». On perçoit toute la subjectivité assumée que suppose cette approche. Dans le même ordre d'idées, Michel Agier interroge et complexifie dans toutes ses recherches ${ }^{9}$ la notion de terrain pour " (remuer) la terre séchée des évidences ${ }^{10}$, remettre en question l'éternelle dichotomie altérité/identité et nous renvoyer à notre humilité : le chercheur dit moins ce qu'il voit que comment il le voit. Dans ce jeu de distanciation, restent finalement le plaisir et le jeu de la rencontre.

\section{La proximité à l'épreuve : deux exemples issus du terrain}

Étant donné que toute méthodologie est constituée à la fois par la démarche du recueil de données et par leur analyse, nous situerons nos discours sur ces deux niveaux. Pour ce faire, nous examinons dans un premier temps deux exemples tirés d'enquêtes "de terrain " par entretiens : la première est extraite d'une étude fondée sur des entretiens sociolinguistiques avec des migrants et la seconde est une recherche-action-formation en didactique des langues où prennent place des entretiens avec des enseignant.e.s. Dans un second temps, nous nous questionnerons sur les enjeux de la "proximité » appliquée à nos domaines de recherche, à savoir la sociolinguistique et la didactique des langues.

\section{L'entretien sociolinguistique et le positionnement du chercheur}

Dans cette section, nous étudions la logique de la proximité en termes de "positionnement du chercheur " par rapport à son objet de recherche et à l'entretien sociolinguistique (notamment la phase de transcription des données et leur analyse).

\section{Définition de l'objet d'étude et problématique}

Pour ce faire, nous abordons le concept de proximité en analysant des extraits issus d'entretiens menés lors d'une enquête de terrain à propos des représentations sociolinguistiques et des connaissances des migrants sur la

7 Notre réflexion sur l'écriture inclusive nous amène à utiliser alternativement le masculin et le féminin pour désigner dans des cas syntaxiques qui accepteraient les deux.

8 François Laplantine, La description ethnographique, Paris, Nathan, 1996, p. 38.

9 Michel Agier, Anthropologues en danger. L'engagement sur le terrain, Paris, éd. Jean-Michel Place, 1997.

10 Michel Agier, La sagesse de l'ethnologue, Paris, L’œil neuf, 2004, p. 6. 
langue italienne et sur les dialectes en Italie pour questionner leur rôle dans les dynamiques d'intégration sociale. ${ }^{11}$ Ces extraits montrent des phénomènes conversationnels inattendus qui se sont vérifiés lors des entretiens et que nous analyserons en tenant compte des réflexions théoriques sur la proximité.

\section{Posture du chercheur}

Rendre compte d'une posture de la proximité revient paradoxalement à se distancier de son objet de recherche afin de comprendre les raisons qui conduisent le chercheur à l'étudier. Cette attitude implique de construire et d'adopter une posture "alter-réflexive " en acceptant " que sa propre histoire sociolangagière fait pleinement partie du répertoire des expériences qui participent à la construction de son regard, de ses interprétations $»^{12}$. Elle demande au chercheur.e intervenant.e "de se considérer comme un élément du "contexte", avec son positionnement personnel, son évolution, son expérience de vie, et à ce titre d'en détailler les paramètres constituants dans sa recherche $» .{ }^{13}$ Autrement dit, l'intérêt et la sensibilité pour un champ d'étude, pour la sociolinguistique, par exemple, ou pour un sujet comme la minoration des langues a sans doute un lien avec le vécu du chercheur. Pour le dire avec Louis-Jean Calvet :

\footnotetext{
"La science n'est jamais neutre, elle est nécessairement compromise, et il nous faut sans cesse jeter un regard épistémologique sur ce que nous faisons. Il ne suffit pas de de nous demander "qu'est-ce que faire de la sociolinguistique?" ou "qu'est-ce que faire de la linguistique ?", il faut sans cesse nous interroger sur la dimension politique de ce que nous faisons, de ce que nous ne faisons pas ou de ce que nous faisons mal $»^{14}$.
}

\section{Le chercheur et le choix de la méthode d'enquête}

Revenons donc au recueil des données, phase centrale de la recherche. Nous prenons en considération l'exemple de l'entretien ; ce dernier est une forme particulière d'interaction où le pouvoir conversationnel des participants est normalement inégalitaire puisque c'est le chercheur qui définit les objectifs et rythme les questions.

11 Les données sur lesquelles nous nous appuyons dans cette contribution sont issues de la thèse doctorat de Valeria Villa, Les dialectes des migrants. Représentations sociolinguistiques et dynamiques d'intégration territoriale dans l'Italie contemporaine, Université Bordeaux Montaigne, 2014.

12 Aude Brétegnier, "Sociolinguistique alter réflexive. Du rapport au terrain à la posture du chercheur ", in Didier De Robillard (éd.), Réflexivité, herméneutique. Vers un paradigme de recherche? Cahiers de Sociolinguistique, ${ }^{\circ}$ 11, Presses Universitaires de Rennes, 2009.

13 Marielle Rispail, "Pouvoir d'agir, intervention ", in Marielle Rispail (éd.), Abécédaire de Sociodidactique. 65 Concepts et notions, Saint-Étienne, PUSE, 2017, p. 102.

14 Louis-Jean Calvet, "Le sociolinguiste et le pouvoir politique ", in Romain Colonna (éd.), Les locuteurs et les langues: pouvoirs, non-pouvoirs et contre-pouvoirs, Limoges, Lambert-Lucas, 2014 , p. 25. 
De ce fait, il est possible que l'informateur ait l'impression de subir un " interrogatoire "- surtout lors de la phase initiale dans l'enchainement de questions d'ordre informatif comme prénom, âge, pays d'origine, etc. - ou de passer presque un " examen " dont il ne possède pas toutes les réponses. Il arrive souvent qu'on entende " je ne sais pas, cela ne me vient pas à l'esprit, je suis désolé $»^{15}$. Néanmoins, il est indispensable de guider l'informateur puisque la réflexion métalinguistique n'est pas un sujet de conversation ordinaire des locuteurs : on entendra difficilement des individus discuter dans la vie courante de leur prononciation des consonnes, des diphtongues, etc. Provoquer ce type de réflexions chez l'informateur devient alors une nécessité pour répondre aux questions de la recherche ${ }^{16}$.

Le chercheur doit donc avoir à cœur de créer un climat détendu et un environnement favorable non seulement à la prise de parole de l'informateur mais surtout à la co-construction de l'entretien qui se manifeste par exemple dans la flexibilité du format de l'interview semi-dirigée. Cela peut déboucher sur des changements dans la répartition de la parole, comme on le verra dans les extraits 1 et 2 analysés plus loin.

\section{Traitement des données}

La question de la "proximité " se pose ensuite lors de la phase de transcription des entretiens sociolinguistiques. On rappellera qu'il s'agit d'une étape importante et non pas d'une simple représentation de l'interaction orale. Comme le souligne Mondada ${ }^{17}$, la chercheure se positionne par rapport à la "politique de la représentation", dans la mesure où

" la question éthique est inséparable des questions scientifiques : en plus du sens qu'ils donnent à leurs expériences, les participants dans le cours de leurs actions construisent aussi leur identité et la transcription est précisément un moyen de la rendre disponible à l'analyse ".

Parmi les questions liées aux politiques de représentation, il y a les choix concernant «l'identification des locuteurs ». Loin d'être une pratique neutre, ces choix entrainent des conséquences sur la catégorisation des locuteurs (âge sexe, profession, classe sociale, rôle institutionnel, etc.). L'identification des participant.e.s à une interaction est l'un des premiers choix du / de la chercheur.e mais aussi la toute première information qui apparait sur la

15 Gabriele Iannaccaro, Il dialetto percepito. Sulla reazione di parlanti di fronte al cambio linguistico, Alessandria, Edizioni dell'Orso, 2002, p. 109.

16 Op. cit., p. 107-108.

17 Lorenza Mondada, "Pratiques de transcriptions et effets de catégorisation ", in Bruno Bonu (éd.), Transcrire l'interaction, Cahiers de praxématique, Université Paul Valery Montpellier, $n^{\circ} 39,2002$, p. 48. 
ligne initiale de la transcription ${ }^{18}$. Cette question, peu problématisée par les analystes de la conversation, est pourtant pertinente en sociolinguistique (et en sociodidactique) puisque décider de l'identification des participant-e-s ou des façons dont on leur donne la parole concerne « les débats sur les marqueurs identitaires des locuteurs et sur leur pertinence pour l'organisation de leurs conduites notamment langagières. ${ }^{19}$ La réflexion sur ce détail descriptif de la transcription, loin d'être marginale, nous rappelle en outre que :

les catégories des participants ne sont pas à définir à priori par l'analyste mais sont construites par les participants dans le déroulement de l'interaction, et par le fait que c'est dans et par l'organisation de distance l'interaction que ces catégories émergent et deviennent pertinentes, contribuant à définir réflexivement le contexte, qui peut lui-même se transformer durant le cours d'action ${ }^{20}$.

Par conséquent, si l'on décide de figer la personne interrogée dans son rôle d'informateur, d'une part on présuppose sans doute qu'il/elle ne pourra pas avoir une autre place dans l'interaction, d'autre part on crée une distance entre celui qui gère l'échange et celui qui le subit.

Or l'extrait que nous allons examiner montre que l'entretien ${ }^{21}$, en tant qu'évènement communicatif, peut au contraire provoquer des situations inattendues. C'est le cas de l'inversion des rôles interviewer/interviewé.e quand on assiste au bouleversement de la structure rigide et asymétrique dans l'attribution des tours de paroles (question-réponse) et dans le temps de parole accordé aux informateurs.

\section{Extrait 1}

Tak. Non so questo è dialetto bolognese o no? Je ne sais pas il s'agit du dialecte bolognais ou pas?

Val. Si si!

Oui oui

Tak. Non so +++ sorbole che mi diceva sempre quando veniva qui e poi::: adesso non mi ricordo che::: +++ ad esempio lei qualcosa qualche dialetto?

Je sais pas +++ "mince " elle me disait toujours quand il venait ici et puis:: maintenant je me souviens pas que::: +++ par exemple vous quelque chose en dialecte?

Val. Ma io::: un po' di dialetto bolognese:::+++ perché io in realtà non sono di Bologna:: io sono [pugliese

18 Op. cit., p. 48.

19 Op. cit., p. 49.

20 Op. cit., p. 57.

21 Conventions de transcription adoptées: Abc. Identification des locuteurs avec trois lettres minuscules en début de ligne ; [ ] chevauchements ; CAPITALE volume fort de la voix ;: prolongement d'un son ;. intonation descendante, conclusive ; , intonation continuée ; ? intonation ascendante ; ! intonation animée ; $\uparrow$ montée et $\downarrow$ descendante intonative ; + pause ; (notes) sur les comportements verbaux et non verbaux; italique mots en dialecte. 
Mais moi ::: je connais peu le dialecte bolognais::: +++ parce que moi en réalité je ne suis pas de Bologne::: je suis [des Pouilles

Tak. Sì::]

Oui:::]

Val. Quindi il dialetto che conosco di più è quello della Puglia::: sai del sud dell'Italia +++ però ho vissuto in questa regione diversi anni +++ ho studiato qui ma adesso ti ho detto sono in Francia:: lavoro a Bordeaux ma è per questo che conosco un po'

Donc le dialecte que je connais davantage est celui des Pouilles::: tu sais celui du sud de l'Italie +++ mais j'ai vécu dans cette région plusieurs années +++ j'ai étudié ici mais maintenant je suis en France comme je te disais je travaille à Bordeaux mais c'est pour cela que je connais un peu

Tak. Sì::: sì:::per questo che hai imparato un po' di dialetto

Oui::: oui::: c'est pour cela que tu as appris un peu de dialecte

Val. Sì capisco [soprattutto

Oui je comprends [surtout

Tak. Ma anche] io::: pure io:::pure io così capisco quando dicono loro ma io diciamo non so quasi niente:::

Mais moi:: aussi] moi aussi::: moi aussi je comprends comme ça quand eux ils disent mais disons que je ne sais presque rien::

Dans le premier tour de cet extrait, l'informateur Tak. devient le metteur en scène de l'interaction en posant d'abord une question pour avoir une confirmation de la chercheure (" non so questo è dialetto bolognese o no? "), puis en l'interrogeant sur sa connaissance des dialectes italiens (" ad esempio lei qualcosa qualche dialetto?"). Durant cette séquence de l'interview, les rôles s'inversent pendant 6 tours et la " chercheure " est interviewée par "l'informateur ». Quelques détails soulignent ce renversement de situation comme l'invitation de Tak. à poursuivre l'explication, le dernier mot du quatrième tour de Tak. "pugliese (ligne 6) " se chevauche avec la réponse si (ligne 7) de Val. Le même phénomène se répète dans les tours suivants (sì:: sì:::per questo che hai imparato un po' di dialetto), jusqu'à donner l'impression d'une conversation informelle sur le thème de la compétence dialectale.

\section{Analyse des données}

Venons-en maintenant à un autre aspect saillant de la méthodologie : quel type d'analyse suppose de rendre compte du concept de proximité ? Interroger la situation d'émergence des récits des informateurs est sûrement une dimension importante car l'entretien est un processus communicatif où les réponses sont difficilement séparables du contexte dans lequel les données ont été produites $^{22}$. Un exemple est proposé par les marqueurs de relation entre le

22 Valeria Villa-Perez, "Les mauvaises langues des migrant.e.s : citoyenneté et migrance en région Émilie-Romagne ", in Vanessa Deluge (éd.), avec la collaboration de Gudrun Ledegen, Mauvaises langues. Migrations et mobilités au cour des politiques, des institutions et des discours, 
langage et l'espace (par exemple «sorbole che mi diceva sempre quando veniva $q u \boldsymbol{q} »)$, les éléments de deixis référés aux lieux professionnels des informateurs où les échanges en dialecte avec des Italiens se sont vérifiés.

Pendant l'entretien, un positionnement de proximité avec son interlocuteur peut aussi favoriser un climat favorable à la prise de parole ; l'échange se transforme en un récit d'épisodes linguistiques ou de vie vécus dans le pays d'accueil ou d'origine. Cet aspect est constitutif de la recherche qualitative puisqu'il permet de faire émerger des singularités, des histoires individuelles, ce qui ne serait pas visible dans une démarche quantitative et statistique.

En guise d'exemple, une question sur la langue d'origine provoque une réponse portant sur les conflits linguistiques entre les Moldaves et les Russes, en Moldavie. Il s'agit de l'entretien avec Rai, une auxiliaire de vie pour personnes âgées, moldave, interrogée à Bologne :

\section{Extrait 2}

Rai.

Da noi sono tanti russi e non vogliono imparare la lingua moldava +++ abitano da cinquanta anni nel nostro paese e no vogliono imparare

Chez nous les Russes sont très nombreux et ils ne veulent pas apprendre la langue moldave +++ ils habitent depuis cinquante ans dans notre pays et ils ne veulent pas apprendre

Val. perché?

\section{Pourquoi?}

Rai. perché? Perché sono loro:::: e quando sono andata io a casa l'anno scorso sono andata al mercato e ho chiesto un paio di scarpe $\downarrow$ eh:: ho provato una scarpa e allora dico::: eh:: mi può dare anche la seconda? In lingua moldava e ho parlato che lei era una signora di cinquantacinque anni russa! $\mathrm{E}$ io dico in lingua moldava e lei fa finta che non mi ha sentito! Io ancora lingua moldava dico:: per piacere mi dai l'altra scarpa? E lei dice::: +++ non capisco e io dico ma come! A questa età? Quanti anni ha lei qui? Ma cosa c'è:: quando vai in Russìa::: eh:: Russia quale lingua parli? In Russia $\downarrow$ A questa età io dico che non sei venuta da ieri in Moldavia ++ sei venuta da trenta quaranta anni! Allora è ora $\downarrow$ per sapere per la lingua nostra!++ e lei non mi ha voluto dare+ la seconda scarpa!

Pourquoi? Parce que c'est eux::: et quant moi j'ai été chez moi l'année dernière je suis allée au marché et j'ai demandé une paire de chaussures $\downarrow$ eh :: jai essayé une chaussure et alors je dis : eh :: peux-tu me donner la deuxième? en langue moldave et j'ai parlé parce quelle était une dame de cinquante cinq ans russe! et moi je dis en langue moldave ET ELLE FAIT COMME SI ELLE NE M'AVAIT PAS ENTENDUE! moi encore langue moldave je dis :: sil te plaît peux-tu me donner l'autre chaussure? et elle dit :: +++ je ne comprends pas et moi je dis mais comment ça! $\grave{A}$ ton âge? ça fait combien d'années que tu es là? mais qu'est-ce qu'il y a :: quant tu vas en

Paris, L'Harmattan, 2018, p. 25-37. 
Russie ::eh :: Russie quelle langue parles-tu ? EN RUSSIE $\downarrow$ À cette âge là moi je dis que tu n'es pas arrivée hier en Moldavie ++ tu es venue depuis trente ou quarante ans! alors c'est le moment $\downarrow$ pour savoir notre langue!++ et ELLE n'a pas voulu me donner + l'autre chaussure!

La demande de clarification ("pourquoi ») sur les raisons qui poussent les Russes à refuser l'apprentissage de la langue moldave déclenche un long récit très animé où Rai. raconte un fait qui s'est produit dans un marché de sa ville. La non compréhension en moldave affichée par son interlocutrice, une commerçante, est interprétée par Rai. comme un refus de parler et de comprendre le moldave.

Un thème apparemment anodin, et non prévu dans le guide d'entretien, peut ainsi permettre au/à la chercheur.e de formuler des hypothèses sur la représentation des langues dominantes/dominées chez un locuteur, quand il sera dans sa phase d'analyse des réponses.

On a voulu montrer, dans la limite de ces quelques exemples, combien une méthodologie de la proximité, qui se veut attentive aux discours qu'elle récolte et à ceux qui les produisent au moins autant qu'aux questions et hypothèses initiales de la chercheure, a des chances de faire surgir des éléments inattendus et féconds pour la recherche. Mais il faut pour cela avoir fait des choix rigoureux dans le dispositif de recherche, choix qui laissent place à l'imprévu, à des trajectoires discursives différentes, à des parenthèses narratives difficilement prévisibles car elles émergent naturellement au fil de la conversation, en essayant "d'instaurer une relation d'écoute active et méthodique, aussi éloignée du pur laisser faire de l'entretien non directif que du dirigisme du questionnaire ${ }^{23}$. Si un telle relation de proximité est instaurée lors de l'interview, on peut essayer de réduire " au maximum la violence symbolique ${ }^{24}$ qui peut $s^{\prime}$ instaurer à travers elle $»^{25}$.

Il s'agit donc des aspects constitutifs de l'enquête de "terrain ", contribuant à la problématisation de cette notion qui

"souffre paradoxalement d'un déficit de théorisation " non seulement en sociolinguistique ${ }^{26}$ mais aussi en sociodidactique alors que " toute recherche sociodidactique commence par étudier la spécificité du terrain où elle s’inscrit, avant de chercher à mettre au jour des corrélations parfois généralisables ou transférables entre les différents paramètres qui la composent. $»^{27}$

23 Pierre Bourdieu, La misère du monde, Paris, Seuil, 1993, p. 1393.

24 C'est nous qui soulignons.

25 Ibid.

26 Didier De Robillard et Marc Debono, «Le sociolinguiste est-il (sur) son terrain? Problématisations d'une métaphore fondatrice ", in Philippe Blanchet et alii (éds), Pluralité linguistique et démarche de recherche. Vers une sociolinguistique complexifiée, Cahiers internationaux de sociolinguistique, $\mathrm{n}^{\circ}$ 2, 2012, Paris, L'Harmattan.

27 Marielle Rispail et Philippe Blanchet, " Principes transversaux pour une sociodidactique dite "de terrain" ", in Philippe Blanchet et Patrick Chardenet (éds), Guide pour la recherche en didac- 


\section{La "proximité »: au cour de la recherche-action-formation ${ }^{28}$ en didactique des langues et des cultures}

Pour notre second exemple, nous commencerons par nous tourner vers le domaine des sciences de l'éducation où la recherche-action a été définie comme une recherche dans laquelle « il faut qu'il y ait à la fois désir de "transformer la réalite" "et mise en place d'un dispositif permettant de produire des connaissances concernant ces transformations" ${ }^{29}$. En poursuivant cette réflexion par rapport à la place et aux objectifs de la recherche-action en didactique des langues et des cultures, Stéphanie Clerc précise :

"La visée [...] n’est pas de généraliser la portée des phénomènes observés mais d'approfondir nos connaissances des effets des pratiques d'enseignement des langues sur l'apprentissage de celles-ci, de transformer des représentations (des langues, de l'apprentissage, de l'enseignement) et d'envisager des transpositions du terrain vers des savoirs pour nourrir ensuite des réflexions sur des transpositions possibles des connaissances mises au jour, moyennant des précautions de recontextualisation incluant les dimensions socio-éducative, économique, politique, idéologique des nouveaux terrains envisagés. ${ }^{30}$ "

Cette précision est particulièrement importante dans le cas de la rechercheaction sur laquelle nous nous penchons ici, car elle affiche clairement une visée formative outre celle de la transformation de la réalité, d'où son appellation de recherche-action-formation.

Par ailleurs, notre réflexion méthodologique sur la "proximité » nous a amenées à avancer vers ce qu'on appelle "la nouvelle recherche-action " ${ }^{31}$ qui « intègre plus largement l'approche ethnographique et les perspectives des acteurs sociaux $^{32}$.»

tique des langues et des cultures. Approches contextualisées, Paris, Les archives contemporaines, 2011, p. 66.

28 Désormais RAF.

29 Jean-François Berthon, « Mode et méthode spécifiques de la recherche en sciences humaines : sa pertinence dans le domaine de la formation et de l'éducation ", document électronique, in Site des Recherches-Innovations de l'Académie de Lille, 2000. Disponible sur http://www2. ac-lille.fr/pasi/bibliographie/recherche-action.pdf).

30 Clerc Stéphanie, Vers une didactique de la pluralité sociolinguistique. Cheminement de la didactique du français langue étrangère à la sociodidactique des langues, Synthèse d'HDR, vol. 1, Université de Provence, 2011, p. 129.

31 D'après William Carr et Stephen Kemmis, in Georges Lapassade, 1993, Familles de rechercheaction. De l'ethnographie de l'école à la nouvelle recherche-action, Document dactylographié, Université de Paris 8.

32 Stéphanie Clerc, "La recherche-action : ancrages épistémologique, méthodologique et éthique ", in Philippe Blanchet et Patrick Chardenet (éds), Guide pour la recherche en didactique des langues et des cultures. Approches contextualisées, Paris, Édition des archives contemporaines, 2015, p. 115. 
Définition de l'objet d'étude et problématique

Que faire des langues et des cultures " autres " rencontrées dans nos classes ? La recherche-action-formation (désormais RAF) L'arc-en-ciel de nos langues - Jalons pour une école plurilingue dont est issu l'ouvrage éponyme a réuni pendant près de 3 ans des enseignant-e-s-chercheur-e-s de l'Université Jean Monnet et des enseignants des $1^{\text {er }}$ et $2^{\text {nd }}$ degrés à Saint-Étienne autour de cette question. L'objectif de la recherche était de récolter, formaliser puis diffuser des pratiques enseignantes à succès dans la mobilisation des langues et des cultures des élèves allophones en classe, afin de mettre ainsi à disposition des enseignant-e-s le travail fait par d'autres enseignant.e.s.

Le choix de la méthode d'enquête

Outre les observations de classes et enregistrements de séquences plurilingues, des moments d'entretiens avec les enseignants pour qu'ils/elles éclaircissent leurs projets et leurs réflexions sur ce qu'ils/elles font en classe ont rythmé le travail de récolte et d'analyse des données. Ces entretiens et extraits d'interactions, ainsi qu'un va-et-vient entre analyses et éclairages théoriques sur la recherche-action nous ont permis, comme nous allons le voir, de mieux saisir la logique et la richesse d'une méthodologie de la proximitésur trois plans : le plan de la relation au "terrain ", le plan méthodologique et celui de la construction de l'objet d'étude.

\section{Terrain et proximité}

Comme dans le cas de toute recherche-action, la «multidimensionalité » du terrain de recherche est la première caractéristique directement liée à la logique de "proximité " lors de la RAF susmentionnée. Elle découle de la relation établie entre trois terrains habituellement étanches : le «terrain » de la recherche étant en même temps celui de la classe de langue et devenant tout en même temps celui de la formation des enseignants de langues. Les traits d'union reliant les termes qui forment l'appellation triangulaire de ce type de recherche : recherche-action-formation, sont par ailleurs un reflet de la contiguité entre ces trois terrains qui se confondent et se fondent pour n'en faire qu'un.

Pour les acteurs participant à la recherche, cette manière de " faire de la recherche " [et] d' "être au terrain ", [permet, par voie de conséquence,] la diffusion des connaissances scientifiques dans le monde social ${ }^{33}$, comme le souligne Stéphanie Clerc, ajoutant ainsi une nouvelle dimension au terrain de la recherche.

33 Stéphanie Clerc, "Recherche-action-formation-intervention ", in Marielle Rispail (éd.), Abécédaire de Sociodidactique. 65 Concepts et notions, Saint-Étienne, PUSE, 2018, p. 109. 
$\mathrm{La}$ " proximité " des terrains facilite les échanges et la circulation entre les acteurs de la recherche qui se traduit, sur le plan méthodologique, par un «brouillage » des rôles et une collaboration accrue et spécifique qui mérite une réflexion particulière.

\section{Place des acteurs/trices-chercheur.e.s et proximité}

Ainsi, il n'y aurait pas de rôles dévolus dès le départ du fait du statut social et professionnel des personnes participant à la recherche. Dans la RAF, L'arcen-ciel de nos langues, tout le monde est chercheur.e et acteur/trice du terrain à la fois. Cette situation peut sembler invraisemblable et trop optimiste, surtout au début, lorsque les participant.e.s arrivent sur le terrain de la recherche avec des rôles sentis comme "inévitablement " prédéterminés du fait de leur statut professionnel. Or, la proximité qui s'établit entre eux/elles au cours de la recherche contribue à modifier cette perception initiale et par conséquent à neutraliser cette hiérarchie supposée de leurs rôles. Ce n'est donc pas une recherche menée sur des personnes, mais avec des personnes, ce qui confere un caractère foncièrement collaboratif à chaque étape de son déroulement :

"(1) une co-observation-description-compréhension du contexte, sur laquelle on revient régulièrement (suite par exemple à l'introduction de nouvelles pratiques) ; (2) une co-construction de l'objet de recherche avec des acteurs du terrain pour (identification de manques ou problèmes perçus) ; (3) une co-élaboration d'une ou d'action(s) innovante(s) pour le terrain investi ;

(4) une co-analyse-évaluation des effets de ce qui a été mis en œuvre. ${ }^{34}$ "

Pour illustrer le travail effectué lors de la « co-observation-descriptioncompréhension du contexte " pendant la RAF qui s'est déroulée à SaintÉtienne, penchons-nous sur trois extraits tirés de deux entretiens réalisés vers la fin de la $1^{\text {re }}$ année : le premier avec un enseignant n'ayant pas participé à la RAF (désigné par E1), le second avec une enseignante ayant participé à la RAF (E2). On peut constater que deux discours opposés sur la variation linguistique émanent de leurs propos :

\section{Extrait 3}

E1. y a des Roms qui parlent le langage de la rue comme par exemple j'm'en fous fiche moi la paix + c'est pas dit méchamment + ils vivent dans la rue ils ont pas de maison donc ils parlent le langage de la rue + ils sont pas censés le savoir je leur dis c'est des bêtises tu peux pas parler comme ça.

E1 affiche une vision étriquée de la variation linguistique qui se résume pour lui à des mots et expressions appartenant à certains registres " extrascolaires " chez les élèves. De bonne volonté, mais non dépourvu d'idées préconçues sur l'origine de ses élèves à qui il impute ce langage de la rue, il 
semble adopter une attitude puriste vis-à-vis de la langue de l'école et sépare les sphères d'activités de différents registres de langue.

E2, au contraire, pose un regard complexe sur la pluralité linguistique en classe et adopte une attitude ouverte à l'égard de la variation linguistique (dans toutes les langues il y a des niveaux de langue /), même si c'est plutôt par obligation professionnelle (je suis obligée + c'est dans les programmes). Malgré une confusion entre " registre " et "variation ", tout au long de l'entretien, elle évolue d'une simple réflexion sur les registres vers l'idée d'une didactisation (des activités plus précises que ça euh ++ je pourrais, je pourrais +++ ah + mais c'est pas une mauvaise idée finalement) de la variation :

\section{Extrait 4}

E2. ce que je fais + je leur dis + je leur dis ++ écoutez ++ enfin ++ pour ceux qui sont un peu plus curieux + bon + vous savez qu'il y a plusieurs niveaux de langue ++ dans votre pays + dans toutes les langues il y a des niveaux de langue+ je vous le dis +je vous le dis + en tant qu'enseignante + voilà ce qu'on dit en langage soutenu + je préfere que vous le sachiez $+(\ldots)$ je le fais + et sans avoir honte quoi ++ non + non + je le dis + sans me dire qu'il faut pas.

E2. dans mon enseignement tu veux dire + j'accorde de l'importance aux registres + je suis obligée + c'est dans les programmes +++ il faut que les élèves connaissent les niveaux de langue + mais je ne consacre pas probablement de séquence sur ça ++ si + je l'ai fait une fois + une séquence à propos de l'interrogation où $j$ 'avais travaillé une fois sur les niveaux de langue + tu vois + familier + standard + soutenu euh $(\ldots)$ des activités plus précises que ça euh ++ je pourrais, je pourrais $+++\mathrm{ah}+$ mais c'est pas une mauvaise idée finalement.

Ce cheminement vers une didactisation de la variation, rendu possible grâce à la proximité entre les différent-e-s acteurs/trice.s de la recherche, s'observe au niveau des analyses du matériau récolté aussi. C'est ainsi que celui-ci fera l'objet d'une modélisation, puis d'une formalisation pour donner lieu enfin à l'ouvrage collectif publié chez L'Harmattan.

\section{Proximité et construction de l'objet d'étude}

La relation avec l'objet d'étude - les langues présentes en classe - s'inscrit sous le signe de la proximité elle aussi. Dans le cadre de la RAF, la proximité a permis aux acteur/trice.s de prendre le contrepied des stéréotypes ambiants en proposant un renversement de regard sur les langues des élèves souvent considérées par les enseignant.e.s comme un problème ou un obstacle pour l'apprentissage du français. Il s'agissait donc de les considérer comme une ressource et une richesse et d'en faire la démonstration.

Un tel changement de regard sur les langues peut se saisir à l'aide des extraits suivants. Le premier est tiré d'entretiens réalisés au début de la RAF. Le second est constitué d'interactions enregistrées lors de la phase de récolte des données dans les classes. Au début, l'idée, selon laquelle l'utilisation des langues d'origine à l'école serait un facteur d'exclusion émerge des propos récoltés : 


\section{Extrait 5}

E4. En récré ? ... plus ils parlent roumain dans la cour, moins ils ont des copains français car ça les exclut des autres...

Notons tout de même que si une tendance dévalorisante se dessine à travers les discours récoltés lors de cette phase, les discours valorisants ne sont pas pour autant absents de la réflexion des enseignant.e.s. De plus, à la fin de la $2^{\mathrm{e}}$ année de la RAF, la tendance semble s'inverser comme en témoignent les interactions enregistrées lors d'une activité portant sur le conte Le Petit Chaperon Rouge en plusieurs langues (l'élève est désigné par A1, l'enseignante par E) :

\section{Extrait 6}

E aujourd'hui nous avons invité $D+$ qui est albanaise comme $G$ et $M+$ qui va raconter l'histoire du Petit Chaperon rouge + c'est une histoire que vous allez écouter en albanais + langue de vos deux copains de classe et que vous vous mettiez du coup à la place de $\mathrm{M}$ et $\mathrm{G}+$ qui ne comprenaient pas du tout le français quand ils sont arrivés en classe au début.

E qu'est-ce qu'il y a dans le panier du petit chaperon rouge en albanais.

A1 une galette et du miel.

E en français on met quoi.

La proximité de la langue de l'autre devient ainsi une occasion pour développer l'empathie et l'ouverture culturelle dans la classe et un objet d'étude pour les acteurs de la RAF.

Comme nous avons tenté de le montrer, la proximité a été au cour des différentes phases de la recherche : elle a permis de construire un corpus complexe et qui prenne en compte l'évolution des acteurs, d'aboutir à des résultats nuancés, d'interroger la place des acteurs/trices dans leur dynamique.

\section{Les enjeux d'une méthodologie de la proximité}

Nous pouvons à présent tirer quelques conclusions sur la pertinence d'une méthodologie de la proximité dans des études portant sur les langues, leurs représentations et leurs contacts. Tout en n'étant que de simples échantillons de plusieurs de nos recherches en cours, il nous semble que les exemples proposés dans notre texte vont dans le sens d'une adéquation féconde du concept de proximité aux études portant sur les phénomènes et répertoires linguistiques. Il rassemble en effet plusieurs facettes de nos démarches et donne une unité à leur diversité :

- en donnant d'abord la parole aux acteurs sociaux au lieu de les figer dans des catégorisations a priori ;

- en respectant, voire en encourageant, la mise au jour de leurs différences au lieu de les écraser au nom de conclusions qu'on souhaite généralisables ;

- en donnant la priorité à l'imprévu plutôt qu'au prévisible dans nos analyses et résultats. 
Ce faisant, deux conséquences méthodologiques apparaissent, qu’il faudrait affiner dans de futures recherches :

- la nécessité de passer la réalité observée au tamis le plus fin possible de notre regard : l'individu, des moments précis de sa vie, le commentaire de micro-évènements, le partage de détails, sans lesquels on risque de passer à côté des méandres et rouages dans lesquels se déroule le fil langagier de nos vies (nous parlions de "loupe " dans notre introduction);

- l'abandon, partiel ou définitif, du recours à des hypothèses de travail, qui préfigurent les résultats à obtenir et risquent de défigurer les discours observés.

Adopter une méthodologie de la proximité suppose alors qu'on accepte d'être étonné par ce qu'on découvre dans l'épaisseur langagière et affective, ou émotionnelle, du corpus étudié, et d'être renvoyé, comme en rebondissant, d'un thème de recherche prévu à un autre imprévu. Une recherche de Marielle Rispail sur des entretiens faits avec R., jeune Marocaine berbère, témoigne de cet étonnement accepté :
"On a ainsi assisté, dans les discours de R. et les commentaires qui les ont accompagnés, à la construction d'un sujet particulier, une jeune fille de 18 ans, à travers ses langues, transmises, enseignées, rencontrées, rêvées. On a pu appré- cier la force d'une parole imagée, réflexive, enracinée, savante parfois dans ses perceptions sans être conceptuelle dans son expression, voire s'étonner du peu d'hésitation de la locutrice, de la longueur de ses répliques, de l'aisance de ses développements qui passent avec souplesse de l'idée à l'exemple et vice-versa. Cela nous amène à nous interroger non sur les propos tenus mais sur les moda- lités de leur émergence : un entretien est un "évènement" interactionnel, au sens du "evenio" latin, ce qui sort, ce qui advient ${ }^{35}$."

L'entretien est comme un moment privilégié, un temps de suspens et d'action où on se demande : que va-t-il se passer ? Pas n'importe quel entretien toutefois! Il reste la voie royale pour réaliser une proximité de principe, surtout si on l'entend dans le sens du philosophe François Jullien :

" (Je crois que) l'entretien a comme fonction de déclore l'un et l'autre, de les déborder et donc de faire que ce qui était au départ l'un et l'autre ne soit plus comme cela. $»^{36}$

Mais on peut aussi se demander si des « enquêtes de proximité " ne pourraient pas inventer d'autres accès à l'autre que ce type de dialogue prévu et préparé. Germaine Tillon disait, faisant le bilan de son travail d'anthropologue, que le meilleur travail d'enquête était peut-être celui où le chercheur se tait et regarde, enregistre, écoute, sans intervenir, le travail de « l'entretien

35 Marielle Rispail, "Les langues c'est une souffrance. Les vicissitudes d'une jeune berbère en France ", in Leila Messaoudi, Marielle Rispail (éds), Des langues minoritaires en contexte plurilingue francophone, Mélanges en hommage à Ahmed Boukous, Cahiers de Linguistique, 42-1, Louvain-La-Neuve, Ėme Éditions, 2016, p. 251.

36 Entretien non publié avec François Jullien réalisé par Claude Fintz à Grenoble. 
muet ", sans question. Sa réflexion ouvre la voie à de nouvelles façons de voir et d'écouter, d'engranger les actions langagières dont nous faisons notre miel, sans les décoller, ou le moins possible, des situations qui leur ont donné naissance, sans les déraciner de leur " terreau », pour reprendre une problématique de notre introduction.

La prudence et la discrétion deviendraient alors les maitres-mots de nos enquêtes, sans lesquelles la proximité risque de nous échapper, au profit d'un de ses simulacres : la conversation vaine ou l'interrogatoire imposé. Si on se donne pour objectif d'être "proche ", de pouvoir agir et observer parce qu'on est proche, on se doit de concevoir aussi des recherches qui ont le temps, qui prennent le temps de la connaissance et de la rencontre. "Apprivoise-moi " disait le renard au Petit Prince. Le temps de l'apprivoisement deviendrait alors le temps fort de toute enquête, la phase nécessaire pour se donner le droit de dire, faire dire ou laisser dire. Il faut peut-être entrer dans un espace-temps ralenti pour mériter la proximité.

C'est vers une "posture de la proximité ", comme dit plus haut, que nous aimerions nous acheminer, posture de l'attente et de la veille, voire d'une vigilance éthique. La fragilité de cette posture lui donne sans doute sa valeur, comme elle pare d'intérêt les détails qu'elle met en lumière, déplaçant parfois l'enjeu de la recherche de son objet de départ vers la richesse des relations établies à son arrivée. Il transforme le travail de recherche en véritable engagement, humain peut-être avant d'être scientifique. Les conditions de cet engagement seraient une autre voie de recherche à explorer et décrire.

L'approche proposée a fort à voir avec la sociodidactique - qui met l'accent sur l'enracinement social et sociolinguistique des questions didactiques - et les approches contextualisées théorisées par Philippe Blanchet pour affirmer combien tout ce qui est dit ne peut se comprendre qu'en rapport étroit avec le tissu humain, relationnel et situationnel qui l'actualise, le chercheur étant compris dans ce contexte. C'est dans une optique parallèle que Marielle Rispail affirme :

"Ce sont donc des discours que le chercheur offre à la société, mais des discours agissants. En effet, dans l'analyse de ses motivations profondes, de ce qui le "meut", le/la chercheur.e ne peut pas occulter ses croyances, convictions, valeurs. ${ }^{37}$

37 Marielle Rispail, "Pouvoir d'agir, intervention ", in Marielle Rispail (éd.), Abécédaire de Sociodidactique. 65 Concepts et notions, Saint-Étienne, PUSE, 2018, p. 102. 
Mais elle a aussi à voir avec les éclairages, jamais trop divers ou nuancés, que peut lui apporter le faisceau convergent des Sciences humaines et de leurs corollaires les plus fins. Ce travail interdisciplinaire n'est pas une des moindres promesses ouvertes par la proximité.

Marielle Rispail

CELEC (EA 3069) et DIPRALANG (EA739) Montpellier 3

Université Lyon UJM Saint-Étienne marielle.rispail@univ-st-etienne.fr

Marine Totozani

CELEC (EA 3069)

Université Lyon UJM Saint-Étienne marine.totozani@univ-st-etienne.fr

Valeria Villa-Perez

CELEC (EA 3069)

Université Lyon UJM Saint-Étienne valeria.villa@univ-st-etienne.fr

\title{
Résumé
}

Dans cette contribution nous présentons des réflexions sur les manifestations des méthodologies de la proximité en sociolinguistique et en sociodidactique des langues. Ces réflexions méthodologiques et épistémologiques s'appuient sur deux recherches : une enquête de terrain réalisée en Italie auprès de migrants adultes et une recherche-action-formation menée dans différentes écoles de la ville de Saint-Étienne avec des enseignants et des élèves allophones nouvellement arrivés.

\section{Mots-clés}

Proximité, approche qualitative, enquête de terrain, recherche-action-formation, migrants.

\begin{abstract}
In this paper we present some considerations on how the "methodologies of proximity" manifest themselves in languages' sociolinguistics and sociodidactics. These methodological and epistemological considerations are based on two studies: a field survey conducted in Italy with adult immigrants and an action research carried out in different schools of Saint-Etienne with teachers and allophone children who had recently arrived in the country.
\end{abstract}

\section{Keywords}

Proximity, qualitative approach, field research, action research, immigrants. 